\title{
Exploring and Expanding the Use of Lexical Chains in Information Retrieval
}

\author{
Technical Report \\ Terry L. Ruas, William I. Grosky \\ University of Michigan - Dearborn, Computer and Information Sciences, Dearborn, USA \\ truas@umich.edu, wgrosky@umich.edu
}

Summary: This technical report explains our advances in the arena of exploring lexical chains construction using WordNet and proposed algorithms for different types of structures.

\section{Best Synset Disambiguation}

The Best Synset Disambiguation is a subroutine that applies and extends the concept of WSD, but considers the synsets extracted from $w_{i}, w_{i-1}$ and $w_{i+1}$. WSD is the problem in which one must decide which sense is better suited for a word in a sentence, given that this word has multiple meanings and each one of them has an influence from other words. We explore this question through the BSID of a word $w_{i}$, which is selected by considering its predecessor $\left(w_{i-1}\right)$, producing a synset called FormerSynsetID $\left(w_{i}\right)\left(\operatorname{FSID}\left(w_{i}\right)\right)$ and its successor $\left(w_{i+1}\right)$ word, producing a synset called LatterSynsetID $\left(w_{i}\right)\left(\operatorname{LSID}\left(w_{i}\right)\right)$ as the algorithm in Fig. 1 illustrates.

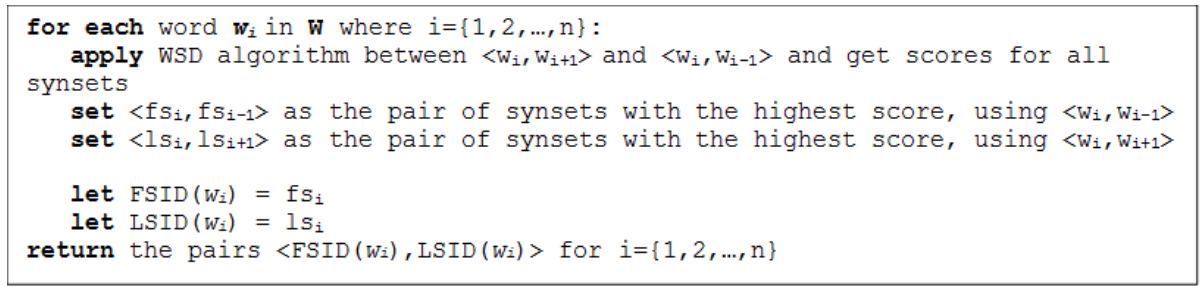

Fig. 1. Former and Latter Synset Selection.

The selection of $\operatorname{FSID}\left(w_{i}\right)$ and $\operatorname{LSID}\left(w_{i}\right)$ is based on the score provided by all possible combinations between all senses of the pairs $\left(w_{i}, w_{i-1}\right)$ and $\left(w_{i}, w_{i+1}\right)$, respectively. This score is calculated applying $\mathrm{Wu} \&$ Palmer's algorithm [1, 2], defined in Eq. 1.

$$
\operatorname{sim}_{W P}\left(c_{1}, c_{2}\right)=\frac{2 * \operatorname{depth}\left(\operatorname{lso}\left(c_{1}, c_{2}\right)\right)}{\operatorname{len}\left(c_{1}, c_{2}\right)+2 * \operatorname{depth}\left(\operatorname{lso}\left(\left(c_{1}, c_{2}\right)\right)\right.}
$$

Where $\operatorname{len}\left(c_{1}, c_{2}\right)$ is the length of the shortest path from synset $c_{1}$ to synset $c_{2}$ in $\mathrm{WN} ; l s o\left(c_{1}, c_{2}\right)$ is the lowest common subsumer of synset $c_{l}$ and synset $c_{2}$; depth $\left(c_{1}\right)$ is the length of the path to synset $c_{l}$ from the root entity (initial synset) in WN. More information about Wu \& Palmer's algorithm can be found in [2], where they conduct a small survey about the most popular WSD algorithms available in the literature.

After each word $w_{i}$ has its own FSID and LSID, it is necessary to find the BSID for this given word $w_{i}$. For this task, we introduce an algorithm called Best Synset Disambiguation (BSD) to suggest the BSID, using as parameters LSID and FSID. Three cases are considered prior to its selection: (a) if $\operatorname{FSID}\left(w_{i}\right)$ and $\operatorname{LSID}\left(w_{i}\right)$ are equal, then $\operatorname{BSID}\left(w_{i}\right)=\operatorname{FSID}\left(w_{i}\right)=\operatorname{LSID}\left(w_{i}\right) ;(b)$ the lowest common subsumer between $\operatorname{FSID}\left(w_{i}\right)$ and $\operatorname{LSID}\left(w_{i}\right)$, given a threshold; and (c), if (b) produces an empty set, the deepest synset among $\operatorname{FSID}\left(w_{i}\right)$ and $\operatorname{LSID}\left(w_{i}\right)$ is chosen. $\operatorname{In}$ case both have the same depth, one is chosen randomly. It is important to mention that, as we traverse the graph in WN for the hypernyms extraction (for each FSID and LSID), we consider the first hypernym in each level, for each synset. Considering that WN organizes the elements in each synset from most to least frequent usage, and we are generalizing the concepts as we move towards the root, it is only natural that we extract a hyerpnym that will provide the most diffused element. Therefore, the first hypernym in every upper level will provide greater probability of an intersection 
with another synset when we build our extended lexical chains. In case both have the same depth, one is chosen randomly. Fig. 2 shows in detail the BSD algorithm.

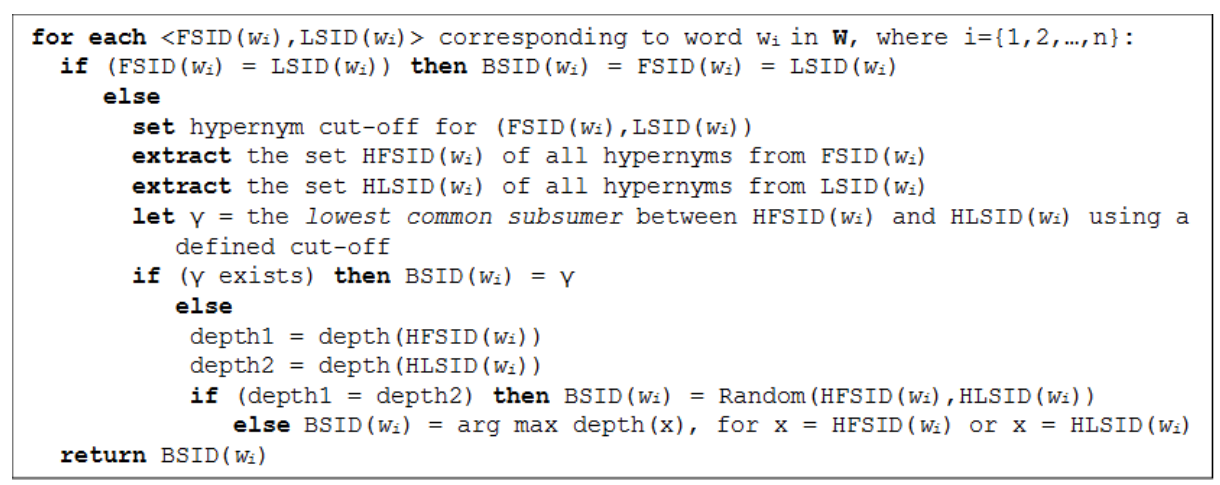

Fig. 2. Best Synset Disambiguation (BSD) Algorithm.

\section{Flexible Lexical Chain Extraction}

Once all words have their BSID selected, we start building our lexical chains in a two-phase subroutine called Lexical Synset Chain Extraction Module. To the best of our knowledge, this module is introducing two novel contributions. First (a), we construct parametrized flexible lexical chains, considering an adaptive structure of synsets based on multilevel hypernyms and second (b), we transform these flexible chains into fixed structures to better represent the semantic values extracted from these synsets.

In (a), we introduce an algorithm called Flexible Lexical Chains (FLC), which extracts these chains, evaluating if a new synset (of a word $w_{i}$ ), or its hypernyms, present lexical cohesion among themselves and the current chain in construction. If the evaluated synset has semantic affinity with the current chain (within a certain level of abstraction in $\mathrm{WN}$ ) this new synset is incorporated to the chain. Otherwise, a new chain will be initialized to capture the next semantic representation.

First, we begin a new FLC inserting the first word, $w_{l}$, from the text into an initial chain structure. Call this first chain FLC(1) and let $\operatorname{BSID}\left(w_{1}\right)$ be the synset id representing the FLC. For each word, $w_{i}$, in the document, where $i=$ $2,3, . ., n$, we verify if $\operatorname{BSID}\left(w_{i}\right)$ is equal to $\operatorname{BSID}\left(w_{i-1}\right)$. If they are the same, we just add $w_{i}$ into the current chain. In case they are not the same, we need to investigate if there is any semantic relationship shared between $\operatorname{BSID}\left(w_{i}\right)$ and $\operatorname{BSID}\left(w_{i-1}\right)$. This is done by extracting all hypernyms from $\operatorname{BSID}\left(w_{i}\right)$ and $\operatorname{BSID}\left(w_{i-1}\right)$, called $\alpha$ and $\beta$ respectively.

Next, we choose the lowest common subsumer, called $\gamma$, between $\alpha$ and $\beta$ given a certain cut-off. This threshold is to avoid that the relatedness between $\alpha$ and $\beta$ is too general, since all synsets in WN are connected by the root entity. If $\gamma$ doesn't exist, it means that $\operatorname{BSID}\left(w_{i}\right)$ and $\operatorname{BSID}\left(w_{i-1}\right)$ are not semantically related in a non-trivial sense, so a new FLC must be initialized. In case $\gamma$ does exist, $w_{i}$ is included into the current FLC and the synset representing the current FLC is updated. Fig. 3 shows in detail each step in the FLC algorithm. 


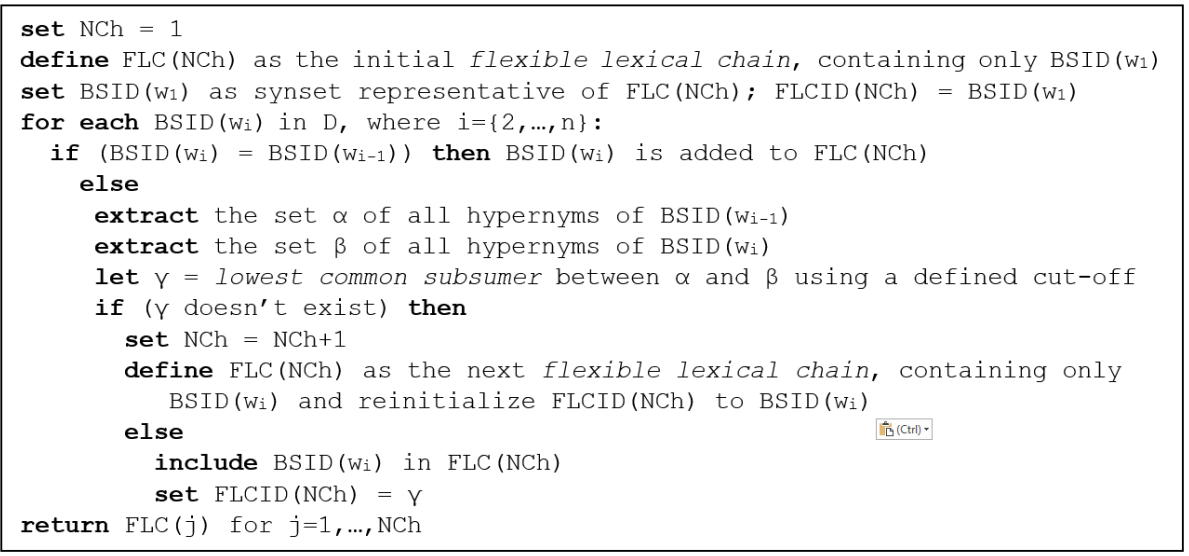

Fig. 3. Flexible Lexical Chain (FLC) Algorithm.

\section{Flexible to Fixed Lexical Chain Extraction (Flex2Fixed)}

After all FLC are produced, we can convert these flexible chains in fixed structures (Flex2Fixed). We assign to all words, $w_{i}$, in the document, the FLCID of the FLC in which this word-synset appears.

For (b) above, after all pairs of words and synsets are arranged, we divide the document into chunks of size $k$ with respect to the number of synsets, represented by $c w_{k}$. Each chunk corresponds to a fixed lexical chain. For each $c w_{k}$, we extract the dominant synset (the one that appears most often in the chunk), called $\theta$, and assign it to represent $c w_{k}$. If there is more than one dominant synset, $\theta$ is chosen randomly. Fig. 4 shows in detail the Flex2Fixed algorithm, while Fig. 5 is a pictorial representation of the process itself. Thus, each fixed lexical chain is represented by a synset. The total number of synsets used in our overall document representation is the union over all documents of the synsets representing all the fixed lexical chains in that document. Call this value NSynsets. Let the synsets used in our document representation be $\operatorname{syn}_{1}, \operatorname{syn}_{2}, \ldots$, syn $_{\text {NSynsets. }}$.

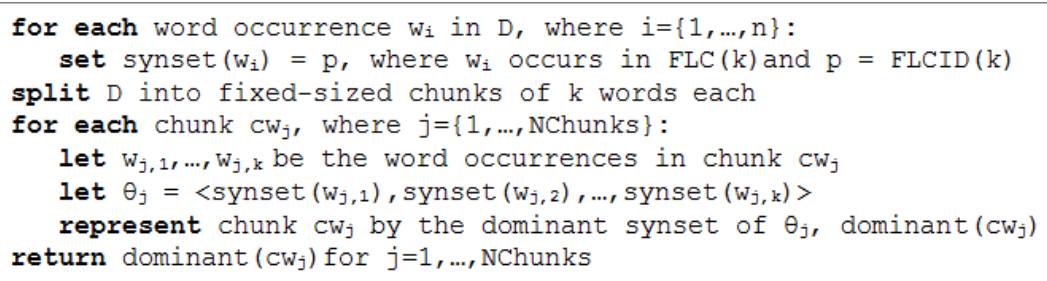

Fig. 4. Flex2Fixed (F2F) Algorithm.

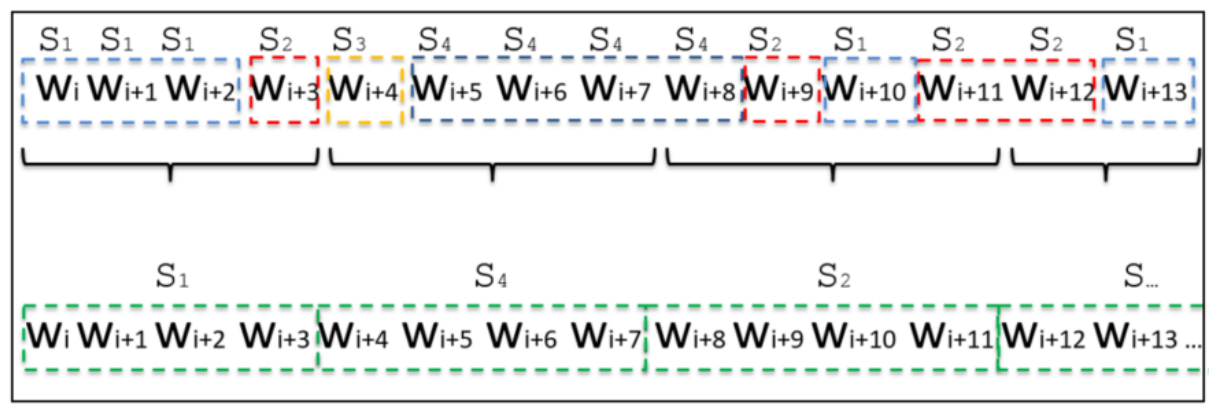

Fig. 5. Flex2Fixed Process. 


\section{Fixed Lexical Chain Extraction}

Once all words have their BSID selected, we can also build fixed chains directly. To the best of our knowledge, there is no previous work in the available literature that constructs parametrized fixed lexical chains for multilevel of hypernyms and chain size.

In this section we introduce an algorithm called Fixed Lexical Chains (FXLC), which extracts these chains given a pre-defined number of synsets that these chains should contain. For each chain $c_{d}$, we extract all hypernyms (including the initial synsets) from all the synsets and call this set $\lambda$. Since each chain must be properly represented, only dominant (appearing in at least half of $c_{d}$ ) synsets, $\beta$, are considered. If there is no dominant synset in the chain, $\lambda$ is selected instead. Let $\delta$ be either $\lambda$ or $\beta$, depending on the preceding condition.

Next, we choose a subset of $\delta$, called $\varepsilon$, of those synsets that are not too close to the root (entity) in WN. This is to prevent providing a synset too general for our chain. If $\varepsilon$ is empty, all synsets in $\delta$ are considered instead. Let $\Omega$ be either $\varepsilon$ or $\delta$, depending on the preceding condition.

Then, from $\Omega$ we extract all maximally occurring synsets, $\alpha$. We then construct $\gamma$, a subset of synsets that occur at the deepest level of $\alpha$. If $\alpha$ has more than one synset, the synset to represent the initial chain $c_{d}$ is then a random synset from $\alpha$. Since we already limit the search in the hypernyms to guarantee a certain level of generality, now we want to maintain the semantic value within each fixed chain. See Fig. 6 for details.

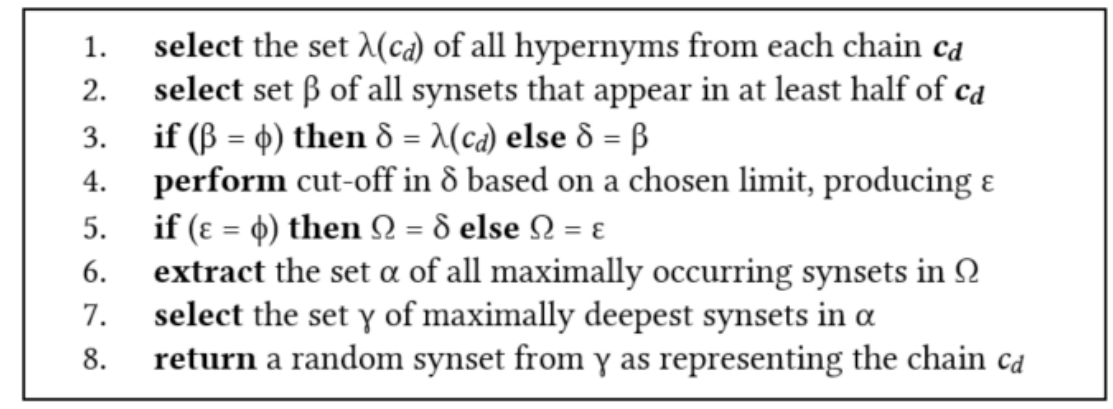

Fig. 6. Fixed Lexical Chains (FXLC) Algorithm.

Thus, each chain is represented by a synset. The total number of synsets used in our document representation is the union over all documents of the synsets representing all the fixed document chains. Call this value NSynsets. Let the synsets used in our document representation be $\operatorname{syn}_{1}, \operatorname{syn}_{2}, \ldots$, syn $_{\mathrm{NSynsets}}$.

Consider document $d$. For each $1 \leq i \leq \mathrm{N}$ Synsets, we define $h(d, i)$ to be the histogram of relative distances between consecutive occurrences of $\operatorname{syn}_{\mathrm{i}}$ in document $d$. Note that the number of bins of $h(d, i)$ and $h(e, j)$ are the same for any 2 documents $d, e$, and synsets $i, j$. Also, for $h(d, i)$, if $\operatorname{syn}_{\mathrm{i}}$ does not occur in document $d$, then the histogram consists of all 0 's. Document $d$ is then represented by the normalized concatenation of $h\left(d\right.$, syn $\left._{1}\right), \ldots, h\left(d\right.$, syn $\left._{N \text { Synsets }}\right)$.

\section{Sample Results of using Lexical Chains for Document Similarity}

This section shows some preliminary results of applying Flexible and Fixed Lexical Chain Extraction in a corpus of 10 webpages from Wikipedia: 05 from dog categories and 05 from computer categories. Each document is used/compared against the entire corpus through the use of cosine similarity, where 1 means a perfect match and 0 the opposite. 


\begin{tabular}{|c|c|c|c|c|c|c|c|c|c|c|}
\hline Flex_BA & DOG A & DOG B & DOG C & DOG D & DOG E & COMP A & COMP B & COMP C & COMP D & COMP E \\
\hline DOG A & 1 & 0.5879 & 0.5932 & 0.6114 & 0.477 & 0.2928 & 0.0775 & 0.2118 & 0.1467 & 0.322 \\
\hline DOG B & 0.5879 & 1 & 0.6825 & 0.7684 & 0.5343 & 0.2119 & 0.0731 & 0.1332 & 0.1582 & 0.1862 \\
\hline DOG C & 0.5932 & 0.6825 & 1 & 0.6895 & 0.5118 & 0.3091 & 0.0945 & 0.1788 & 0.2272 & 0.3038 \\
\hline DOG D & 0.6114 & 0.7684 & 0.6895 & 1 & 0.5575 & 0.1623 & 0.0518 & 0.109 & 0.1053 & 0.1514 \\
\hline DOG E & 0.477 & 0.5343 & 0.5118 & 0.5575 & 1 & 0.2076 & 0.0564 & 0.158 & 0.1085 & 0.2275 \\
\hline COMP A & 0.2928 & 0.2119 & 0.3091 & 0.1623 & 0.2076 & 1 & 0.3827 & 0.7446 & 0.6829 & 0.7553 \\
\hline COMP B & 0.0775 & 0.0731 & 0.0945 & 0.0518 & 0.0564 & 0.3827 & 1 & 0.2754 & 0.6658 & 0.2273 \\
\hline COMP C & 0.2118 & 0.1332 & 0.1788 & 0.109 & 0.158 & 0.7446 & 0.2754 & 1 & 0.4557 & 0.6547 \\
\hline COMP D & 0.1467 & 0.1582 & 0.2272 & 0.1053 & 0.1085 & 0.6829 & 0.6658 & 0.4557 & 1 & 0.475 \\
\hline COMP E & 0.322 & 0.1862 & 0.3038 & 0.1514 & 0.2275 & 0.7553 & 0.2273 & 0.6547 & 0.475 & \\
\hline
\end{tabular}

Fig. 7. FLC document similarity considering the relative distance of chains.

\begin{tabular}{|c|c|c|c|c|c|c|c|c|c|c|}
\hline Flex_TA & DOG A & DOG B & DOG C & DOG D & DOG E & COMP A & COMP B & COMP C & COMP D & COMP E \\
\hline DOG A & 1 & 0.2971 & 0.3265 & 0.3377 & 0.3245 & 0.1933 & 0.0944 & 0.1561 & 0.1319 & 0.2265 \\
\hline DOG B & 0.2971 & 1 & 0.2659 & 0.33 & 0.305 & 0.1253 & 0.0914 & 0.1431 & 0.1487 & 0.1217 \\
\hline DOG C & 0.3265 & 0.2659 & 1 & 0.4186 & 0.3456 & 0.2151 & 0.0876 & 0.1411 & 0.1438 & 0.2356 \\
\hline DOG D & 0.3377 & 0.33 & 0.4186 & 1 & 0.3361 & 0.1681 & 0.0859 & 0.1514 & 0.1032 & 0.1732 \\
\hline DOG E & 0.3245 & 0.305 & 0.3456 & 0.3361 & 1 & 0.1751 & 0.0967 & 0.1498 & 0.1224 & 0.1975 \\
\hline COMP A & 0.1933 & 0.1253 & 0.2151 & 0.1681 & 0.1751 & 1 & 0.2809 & 0.3624 & 0.3158 & 0.4622 \\
\hline COMP B & 0.0944 & 0.0914 & 0.0876 & 0.0859 & 0.0967 & 0.2809 & 1 & 0.2178 & 0.3808 & 0.1865 \\
\hline COMP C & 0.1561 & 0.1431 & 0.1411 & 0.1514 & 0.1498 & 0.3624 & 0.2178 & 1 & 0.2259 & 0.3375 \\
\hline COMP D & 0.1319 & 0.1487 & 0.1438 & 0.1032 & 0.1224 & 0.3158 & 0.3808 & 0.2259 & 1 & 0.2392 \\
\hline COMP E & 0.2265 & 0.1217 & 0.2356 & 0.1732 & 0.1975 & 0.4622 & 0.1865 & 0.3375 & 0.2392 & \\
\hline
\end{tabular}

Fig. 8. FLC document similarity considering the absolute position of chains.

\begin{tabular}{|c|c|c|c|c|c|c|c|c|c|c|}
\hline \multicolumn{11}{|c|}{ FIXED Algorithm } \\
\hline Fixed_BA & DOG A & DOG B & DOG C & DOG D & DOG E & COMP A & COMP B & COMP C & COMP D & COMP E \\
\hline DOG A & 1 & 0.6328 & 0.8718 & 0.7707 & 0.8244 & 0.5644 & 0.2876 & 0.2932 & 0.3438 & 0.488 \\
\hline DOG B & 0.6328 & 1 & 0.719 & 0.7119 & 0.6457 & 0.3222 & 0.193 & 0.1374 & .2587 & 0.2342 \\
\hline DOG C & 0.8718 & 0.719 & 1 & 0.8715 & 0.8308 & 0.6518 & 0.3488 & 0.337 & 0.4942 & 0.5267 \\
\hline DOG D & 0.7707 & 0.7119 & 0.8715 & 1 & 0.8284 & 0.3772 & 0.1975 & 0.1912 & 0.2863 & 0.2949 \\
\hline DOG E & 0.8244 & 0.6457 & 0.8308 & 0.8284 & 1 & 0.5147 & 0.2578 & 0.2876 & 0.3518 & 0.4282 \\
\hline COMP A & 0.5644 & 0.3222 & 0.6518 & 0.3772 & 0.5147 & 1 & 0.4774 & 0.7281 & 0.6953 & 0.9093 \\
\hline COMP B & 0.2876 & 0.193 & 0.3488 & 0.1975 & 0.2578 & 0.4774 & 1 & 0.3529 & 0.4712 & 0.4186 \\
\hline COMP C & 0.2932 & 0.1374 & 0.337 & 0.1912 & 0.2876 & 0.7281 & 0.3529 & 1 & 0.3744 & 0.8445 \\
\hline COMP D & 0.3438 & 0.2587 & 0.4942 & 0.2863 & 0.3518 & 0.6953 & 0.4712 & 0.3744 & 1 & 0.5194 \\
\hline COMP E & 0.488 & 0.2342 & 0.5267 & 0.2949 & 0.4282 & 0.9093 & 0.4186 & 0.8445 & 0.5194 & 1 \\
\hline
\end{tabular}

Fig. 9. FXLC document similarity considering the relative distance of chains. 


\begin{tabular}{|l|rrrrrrrrrrr|}
\hline Fixed_TA & DOG A & \multicolumn{1}{c}{ DOG B } & \multicolumn{1}{c}{ DOG C } & \multicolumn{1}{c}{ DOG D } & \multicolumn{1}{c}{ DOG E } & \multicolumn{1}{c}{ COMP A } & \multicolumn{1}{c}{ COMP B } & \multicolumn{1}{c}{ COMP C } & COMP D & COMP E \\
\hline DOG A & 1 & 0.3243 & 0.4965 & 0.4484 & 0.5173 & 0.3659 & 0.2168 & 0.2613 & 0.2335 & 0.3374 \\
DOG B & 0.3243 & 1 & 0.3473 & 0.3626 & 0.356 & 0.2192 & 0.1579 & 0.155 & 0.1941 & 0.1907 \\
DOG C & 0.4965 & 0.3473 & 1 & 0.6274 & 0.5414 & 0.435 & 0.2671 & 0.2757 & 0.3004 & 0.4398 \\
DOG D & 0.4484 & 0.3626 & 0.6274 & 1 & 0.5167 & 0.3187 & 0.1827 & 0.2282 & 0.2354 & 0.2882 \\
DOG E & 0.5173 & 0.356 & 0.5414 & 0.5167 & 1 & 0.3532 & 0.2067 & 0.2383 & 0.2907 & 0.3034 \\
COMP A & 0.3659 & 0.2192 & 0.435 & 0.3187 & 0.3532 & 1 & 0.3705 & 0.5108 & 0.4603 & 0.6438 \\
COMP B & 0.2168 & 0.1579 & 0.2671 & 0.1827 & 0.2067 & 0.3705 & 1 & 0.3215 & 0.3586 & 0.3514 \\
COMP C & 0.2613 & 0.155 & 0.2757 & 0.2282 & 0.2383 & 0.5108 & 0.3215 & 1 & 0.3575 & 0.585 \\
COMP D & 0.2335 & 0.1941 & 0.3004 & 0.2354 & 0.2907 & 0.4603 & 0.3586 & 0.3575 & 1 & 0.383 \\
COMP E & 0.3374 & 0.1907 & 0.4398 & 0.2882 & 0.3034 & 0.6438 & 0.3514 & 0.585 & 0.383 & 1 \\
\hline
\end{tabular}

Fig. 10. FXLC document similarity considering the absolute position of chains.

\section{References}

1. Wu, Z., Palmer, M.: Verb semantics and lexical selection. 32nd Annu. Meet. Assoc. Comput. Linguist. 133138 (1994).

2. Meng, L., Huang, R., Gu, J.: A Review of Semantic Similarity Measures in WordNet. Int. J. Hybrid Inf. Technol. 6, 1-12 (2013). 